\title{
Feeling backwards? How temporal order in speech affects the time course of vocal emotion recognition
}

\author{
Simon Rigoulot ${ }^{1,2 *}$, Eugen Wassiliwizky ${ }^{1,3}$ and Marc D. Pell ${ }^{1,2}$ \\ 1 Faculty of Medicine, School of Communication Sciences and Disorders, McGill University, Montreal, OC, Canada \\ ${ }^{2}$ McGill Centre for Research on Brain, Language and Music, Montreal, OC, Canada \\ ${ }^{3}$ Cluster of Excellence "Languages of Emotion," Freie Universität Berlin, Berlin, Germany
}

Edited by:

Anjali Bhatara, Université Paris

Descartes, France

Reviewed by:

David V. Becker, Arizona State

University, USA

Emiel Krahmer, Tilburg University,

Netherlands

\section{*Correspondence:}

Simon Rigoulot, Faculty of

Medicine, School of Communication

Sciences and Disorders, McGill

University, 1266 Avenue des Pins

Ouest, Montreal, OC H3G 1A8,

Canada

e-mail:simon.rigoulot@

mail.mcgill.ca
Recent studies suggest that the time course for recognizing vocal expressions of basic emotion in speech varies significantly by emotion type, implying that listeners uncover acoustic evidence about emotions at different rates in speech (e.g., fear is recognized most quickly whereas happiness and disgust are recognized relatively slowly; Pell and Kotz, 2011). To investigate whether vocal emotion recognition is largely dictated by the amount of time listeners are exposed to speech or the position of critical emotional cues in the utterance, 40 English participants judged the meaning of emotionally-inflected pseudo-utterances presented in a gating paradigm, where utterances were gated as a function of their syllable structure in segments of increasing duration from the end of the utterance (i.e., gated syllable-by-syllable from the offset rather than the onset of the stimulus). Accuracy for detecting six target emotions in each gate condition and the mean identification point for each emotion in milliseconds were analyzed and compared to results from Pell and Kotz (2011). We again found significant emotion-specific differences in the time needed to accurately recognize emotions from speech prosody, and new evidence that utterance-final syllables tended to facilitate listeners' accuracy in many conditions when compared to utterance-initial syllables. The time needed to recognize fear, anger, sadness, and neutral from speech cues was not influenced by how utterances were gated, although happiness and disgust were recognized significantly faster when listeners heard the end of utterances first. Our data provide new clues about the relative time course for recognizing vocally-expressed emotions within the 400-1200 ms time window, while highlighting that emotion recognition from prosody can be shaped by the temporal properties of speech.

Keywords: vocal emotions, prosody, speech perception, auditory gating, acoustics

\section{INTRODUCTION}

Emotional events, and more specifically social displays of emotion-the expression of a face, the tone of a speaker's voice, and/or their body posture and movements-must be decoded successfully and quickly to avoid negative outcomes and to promote individual goals. Emotional expressions vary according to many factors, such as their mode of expression (auditory/visual), valence (positive/negative), power to arouse (low/high), antecedents, and potential outcomes (see Scherer, 2009 for a discussion). As early as the seventeenth century, these differences raised the question of the specificity of emotions; in his Traité "Les Passions de l'Ame," the French philosopher Descartes proposed the existence of six "primary" emotions from which all other emotions are derived. In recent decades, studies demonstrating accurate pan-cultural recognition of emotional faces (Izard, 1971; Ekman, 1972) and distinct patterns of autonomic nervous system activity in response to certain emotions (e.g., Ekman et al., 1983; Levenson, 1992) have served to fuel the idea of a fixed set of discrete and hypothetically "basic" emotions, typically anger, fear, disgust, sadness, and happiness, although opinions vary (see Ekman, 1992; Sauter et al., 2010).
Within this theoretical framework, expressions of basic emotion possess unique physical characteristics that render them discrete in communication when conveyed in the face as well as in the voice (Ekman, 1992), although the vast majority of this work has focused on communication in the facial channel.

The structure of vocal emotion expressions embedded in spoken language, or emotional prosody, is now being investigated systematically from different perspectives. Perceptual-acoustic studies show that basic emotions can be reliably identified and differentiated at high accuracy levels from prosodic cues alone, and that these expressions are marked by distinct acoustic patterns characterized by differences in perceived duration, speech rate, intensity, pitch register and variation, and other speech parameters (among many others, Cosmides, 1983; Scherer et al., 1991; Banse and Scherer, 1996; Sobin and Alpert, 1999; Johnstone and Scherer, 2000; Juslin and Laukka, 2003; Laukka et al., 2005; Pell et al., 2009). For example, speech rate tends to decrease when speakers are sad and increase when speakers experience fear; at the same time, differences in relative pitch height, variation, and other cue configurations serve to differentiate these (and other) emotional meanings (see Juslin and Laukka, 2003 for 
a comprehensive review). Similar to observations in the visual modality, cross-cultural studies on the identification of vocal emotions show that anger, fear, sadness, happiness, and disgust can be recognized by listeners at levels significantly above chance when they hear semantically-meaningless "pseudo-utterances" or utterances spoken in a foreign language (Scherer et al., 2001; Thompson and Balkwill, 2006; Pell et al., 2009; Sauter et al., 2010). These data argue that basic emotions conveyed by speech prosody exhibit a core set of unique physical/acoustic properties that are emotion-specific and seemingly shared across languages (Scherer et al., 2001; Pell et al., 2009).

A critical process that has been underestimated in the characterization of how vocal emotions are communicated is the time course for recognizing basic emotions in speech. In the visual modality, the time course for recognizing emotional facial expressions has been investigated by presenting static displays of facial expressions (Tracy and Robins, 2008) or animated face stimuli (Becker et al., 2012). In this latter study, the authors used a morphed continuum running from a neutral exemplar to either a happy or an angry expression and found that happy faces were recognized faster than angry faces, suggesting temporal specificities in the process for recognizing basic emotions in the visual modality (see Palermo and Coltheart, 2004). Since emotional meanings encoded by prosody can only be accessed from their temporal acoustic structure, it is surprising that comparative data on the time course for recognizing basic emotions from prosody remain sparse.

Recently, two studies (Cornew et al., 2010; Pell and Kotz, 2011) examined the temporal processing of vocal emotion expressions using a modified version of Grosjean's (1980) gating paradigm. The auditory gating procedure-originally designed to pinpoint how much acoustic information is needed for lexical access and word recognition-consists of artificially constructing "gates" as a function of specific time increments or of relevant linguistic units of spoken language; the gated stimuli are judged by listeners in blocks of increasing gate duration, typically starting at the onset of the relevant stimulus, where the last gate presented usually corresponds to the entire stimulus event (see Grosjean, 1996 for a discussion of methodological variables). An emotional variant of this paradigm considers how much acoustic information is needed for vocal emotions to be registered and consciously accessed for explicit recognition, using a forced-choice emotionlabeling paradigm. Given the hypothesis that acoustic patterns reflect "natural codes" that progressively activate stored conceptual information about basic emotions (e.g., Schirmer and Kotz, 2006; Wilson and Wharton, 2006), this emotional gating procedure allows inferences about the time course of emotion processing in the specific context of speech, and whether the time needed varies as a function of the emotional signal being transmitted.

In the first study, Cornew and colleagues (2010) presented English-like pseudo-utterances spoken in a happy, angry, or neutral prosody to English listeners spliced into 250 millisecond (ms) gates of increasing duration. Following each stimulus, participants made a three-choice forced response to identify the meaning conveyed. The authors found that listeners required less time (i.e., exposure to acoustic information) to identify neutral sentences when compared to angry and happy sentences, suggesting that vocal emotion expressions unfold at different rates (an effect the authors attributed to a neutral bias in perception). The idea that vocal emotions unfold at different rates was replicated by Pell and Kotz (2011), who gated English-like pseudo-utterances as a function of their syllable structure as opposed to specific time increments. Forty-eight English participants listened to 7syllable utterances conveying one of five basic emotions (anger, disgust, fear, sadness, happiness) or neutral prosody, beginning with presentation of only the first syllable of the utterance, the first two syllables, and so forth until the full sentence was presented (a six-choice forced response was recorded). Emotion identification times were then calculated by converting the number of syllables needed to accurately identify the target emotion of each utterance without further changes in the participant's response at longer gate intervals, into their actual duration for recognition.

Results showed that there were important emotion-specific differences in the accuracy and time course for recognizing vocal emotions, with specific evidence that fear, sadness, neutral, and anger were recognized from significantly less acoustic information than happiness or disgust, from otherwise identical pseudo-utterances. Prosodic cues conveying neutral, fear, and sadness and anger could be detected from utterances lasting approximately $500-700 \mathrm{~ms}(M=510,517,576$, and $710 \mathrm{~ms}$, respectively), whereas happiness $(M=977 \mathrm{~ms})$ and disgust $(M=$ $1486 \mathrm{~ms}$ ) required substantially longer stimulus analysis. Despite the fact that Cornew et al. (2010) focused on a restricted set of emotions when compared to Pell and Kotz (3-choice vs. 6choice task), and gated their stimuli in a different manner $(250 \mathrm{~ms}$ increments vs. syllables), there were notable similarities between the two studies in the average times needed to identify neutral (444 vs. $510 \mathrm{~ms}$ ), angry (723 vs. $710 \mathrm{~ms}$ ), and happy expressions (802 vs. 977 ms, respectively), although Pell and Kotz's (2011) results show that this does not reflect a bias for recognizing neutral prosody as initially proposed (Cornew et al., 2010). Together, these studies establish that the time course of vocal emotion recognition in speech varies significantly according to the emotional meaning being conveyed, in line with results demonstrating emotion-specificity in facial emotion recognition (Becker et al., 2012), although the relative pattern of emotion-specific differences observed in the auditory vs. visual modality appears to be quite different as noted elsewhere in the literature using different experimental paradigms (e.g., Wallbott and Scherer, 1986; Paulmann and Pell, 2011).

Of interest here, closer inspection of Pell and Kotz's (2011) data reveal that recognition of happiness and disgust, in contrast to other basic emotions, improved at relatively long utterance durations (5-7 syllables); in fact, when full sentences were presented, recognition of happy prosody was comparable in accuracy to sadness, anger, and fear despite the fact that these latter emotions were recognized much more accurately than happiness following brief stimulus exposure. Some emotions such as happiness and fear seemed to be particularly salient when the last syllables were presented, leading to significant increases in recognition accuracy at the end of utterances in that study. These results imply that the amount of time needed to identify basic emotions from prosody 
depends partly on the position of salient acoustic properties in speech, at least for certain emotions. Interestingly, Pell (2001) reported that happy utterances exhibit unique acoustic differences in sentence-final position when compared to linguistically identical angry, sad, and neutral utterances, arguing that the position of acoustic cues, and not just time, is a key factor in communicating vocal emotions in speech. Other data underscore that the ability to recognize basic emotions varies significantly depending on the channel of expression-i.e., whether conveyed by facial expressions, vocal expressions, or linguistic content (Paulmann and Pell, 2011) — with evidence that fear, sadness, anger, and neutral are effectively conveyed by speech prosody, whereas other emotions such as happiness or disgust are much more salient in other channels (Paulmann and Pell, 2011). These findings raise the possibility that when basic emotions are preferentially communicated in channels other than the voice, vocal concomitants of these emotions are encoded and recognized somewhat differently; for example, they could be partly marked by local variations in acoustic cues that signal the interpersonal function or social relevance of these cues to the listener at the end of a discourse, similar to how the smile may reflect happiness or may serve social functions such as appeasement or dominance (Hess et al., 2002).

Further investigations are clearly needed to understand the time course of vocal emotion recognition in speech and to inform whether temporal specificities documented by initial studies (Cornew et al., 2010; Pell and Kotz, 2011) are solely dictated by the amount of time listeners require to identify vocal emotions, or whether linguistic structure plays a role for identifying some emotions. We tested this question using the same gating paradigm and emotionally-inflected utterances as Pell and Kotz (2011), although here we presented pseudo-utterances gated syllable-bysyllable from the offset rather than the onset of the stimulus (i.e., in a "backwards" or reverse direction) to test whether recognition times depend on how utterances are presented. If the critical factor for recognizing certain basic emotions in the voice is the unfolding of acoustic evidence over a set period of time, we expected similar outcomes/emotion identification times as those reported by Pell and Kotz (2011) irrespective of how utterances were gated; this result would establish that modal acoustic properties for understanding emotions tend to permeate the speech signal (perhaps due to their association with distinct physiological "push effects," e.g., Scherer, 1986, 2009) and are decoded according to a standard time course. However, if important acoustic cues for recognizing vocal emotions are differentially encoded within an utterance, we should witness significantly different emotion identification times here when utterances are gated from their offset when compared to when they are presented from their onset (Pell and Kotz, 2011). This result could supply evidence that some emotions are "socialized" to a greater extent in the context of speech prosody through functionally distinct encoding processes.

\section{METHODS \\ PARTICIPANTS}

Forty native English speakers recruited through campus advertisements ( 20 men/20 women, mean age: $25 \pm 5$ years) took part in the study. All participants were right-handed and reported normal hearing and normal or corrected-to-normal vision. Informed written consent was obtained from each participant prior to the study which was ethically approved by the Faculty of Medicine Institutional Review Board at McGill University (Montréal, Canada). Before the experiment, each participant completed a questionnaire to establish basic demographic information (age, education, language skills).

\section{STIMULI}

As described by Pell and Kotz (2011), the stimuli were emotionally-inflected pseudo-utterances (e.g., The placter jabored the tozz) selected from an existing database of recorded exemplars, validated and successfully used in previous work (e.g., Pell et al., 2009; Paulmann and Pell, 2010; Rigoulot and Pell, 2012). Pseudo-utterances mimic the phonotactic and morpho-syntactic properties of the target language but lack meaningful lexicalsemantic cues about emotion, allowing researchers to study the isolated effects of emotional prosody in speech (see Scherer et al., 1991; Pell and Baum, 1997 for earlier examples). The selected utterances were digitally recorded by two male and two female speakers in a sound-attenuated booth, saved as individual audio files, and perceptually validated by a group of 24 native listeners using a seven forced-choice emotion recognition task (see Pell et al., 2009, for full details). For this study we selected a subset of 120 pseudo-utterances that reliably conveyed anger, disgust, fear, happiness, sadness and neutral expressions to listeners (20 exemplars per emotion). Thirteen unique pseudo-utterance phrases produced by the four speakers to convey each emotion were repeated throughout the experiment (see Section Appendix). These sentences were the same in their (pseudo) linguistic content as those presented by Pell and Kotz (2011), although the precise recordings selected here were sometimes different because some phrases were emotionally expressed by a different speaker (75\% of the chosen recordings were identical to those presented by Pell and Kotz, 2011). For all emotions, the target meaning encoded by prosody for these items was recognized at very high accuracy levels based on data from the validation study (anger $=$ $86 \%$; disgust $=76 \%$; fear $=91 \%$; happiness $=84 \%$; sadness $=$ $93 \%$; neutral $=83 \%$, where chance in the validation study was approximately $14 \%$ ). Pseudo-utterances conveying each emotion were produced in equal numbers by two male and two female speakers and were all seven syllables in length prior to gate construction.

\section{GATE CONSTRUCTION}

Each utterance was deconstructed into seven gates according to the syllable structure of the sentence using Praat speech analysis software (Boersma and Weenink, 2012). As we were interested in the time course of emotion recognition when utterances were presented from their end to their beginning, the first Gate (Gate_7) of each stimulus consisted of only the last syllable of the utterance, the second gate (Gate_6-7) consisted of the last two syllables, and so on to Gate_1-7 (presentation of the full utterance). For each of the 120 items, this procedure produced seven gated stimuli (Gate_7, Gate_6-7, Gate_5-7, Gate_4-7, Gate_3-7, Gate_2-7, Gate_1-7) each composed of a different number of 
syllables $(120 \times 7=840$ unique items $)$. Note that since the onset of most gated stimuli occurred at a syllable break within the utterance (with the exception of Gate_1-7), these stimuli gave the impression of being "chopped off" at the beginning and starting abruptly. As shown in Table 1, the duration of items presented in each gate condition differed by emotion type due to welldocumented temporal differences in the specification of vocal emotion expressions (Juslin and Laukka, 2003; Pell and Kotz, 2011).

\section{EXPERIMENTAL DESIGN/PROCEDURE}

Participants were invited to take part in a study of "communication and emotion"; they were seated in a quiet, dimly lit room at a $75 \mathrm{~cm}$ distance from a laptop screen. SuperLab 4.0 software (Cedrus, USA) was used to present auditory stimuli played over volume-adjustable, high-quality headphones.

Seven presentation blocks were built, each containing 120 items with the same gate duration (i.e., number of syllables) presented successively in blocks of increasing syllable duration. The first block contained all Gate_7 stimuli (tokens with only the last syllable), the second block contained all Gate_6-7 stimuli (last two syllables), and so on until the Gate_1-7 block containing the full utterances was presented. As in Pell and Kotz (2011), this block design was chosen to mitigate potential artifacts such as response perseveration (Grosjean, 1996). Individual stimuli were randomized within blocks, and participants were instructed to identify the emotion expressed by the speaker as accurately and quickly as possible from six alternatives presented on the computer screen (anger, disgust, fear, sadness, happiness, neutral). Responses were recorded by a mouse click on the corresponding emotion label. Following the emotion response, a new screen appeared asking participants to rate how confident they were about their emotional decision along a 7-point scale, where 1 indicated they were "very unsure" and 7 meant that they were "very sure" about their judgment. After recording the confidence rating, a gap of $2 \mathrm{~s}$ separated the onset of the next trial.

Participants completed ten practice trials at the beginning of the testing session and additional practice trials prior to each block to become familiar with stimuli representing each gate duration condition. Participants were allowed to adjust the volume during the first practice block of each session. Since the volume of our stimuli was homogenized, only one adjustment at the beginning was necessary to meet the participants' individual preferences. The full experiment was administered during two separate 60-min sessions (session $1=$ first three gate conditions, session $2=$ last four gate conditions) to reduce fatigue and familiarity with the stimuli. Participants received \$25 CAD compensation for their involvement.

\section{STATISTICAL ANALYSES}

Participants' ability to identify emotional target meanings (\% correct) and their associated confidence ratings (7-pt scale) were each analyzed. From the uncorrected accuracy (hit) rates of each participant, Hu-scores were computed for each gate and emotion to adjust for individual response biases when several emotion categories are used (see Wagner, 1993). The computation of Huscores takes into account how many stimulus categories and answer possibilities are given in the forced choice task. If only two stimulus categories and two answer possibilities are used (e.g., neutral and anger) the Hu-score for the correct identification of one category, say anger, would be computed as follows: $H u=a / a+b \times a / a+c$. Here $a$ is the number of correctly identified stimuli (anger was recognized as anger), $b$ is the number of misidentifications, in which anger was incorrectly labeled as neutral, whereas $c$ is the number of misidentifications, in which neutral was incorrectly labeled as anger. Wagner (1993) describes the Hu-scores as "[...] the joint probability that a stimulus category is correctly identified given that it is presented at all and that a response is correctly used given that it is used at all."

Hu-scores and confidence scores were submitted to separate $7 \times 6$ ANOVAs with repeated measures of gate duration (seven levels) and emotion (anger, disgust, fear, happiness, sadness, neutral). To infer how much time participants required to correctly identify emotions, we computed the "emotion identification point" for each of the 120 pseudo-utterances by determining the gate condition where a participant identified the target emotion without subsequent changes at longer gate durations of the same stimulus. The emotion identification points were then transformed into "emotion identification times" by converting the number of syllables needed to identify the target into the exact speech duration in milliseconds, which was then averaged across items for each participant (see Pell and Kotz, 2011 for detailed procedures). Of the 4800 possible identification points $(20$ items $\times 6$ emotions $\times 40$ participants $), 419$ items that were not correctly identified by a participant even when the

Table 1 | Duration of the stimuli presented in the experiment in each gate duration condition as a function of emotion.

\begin{tabular}{|c|c|c|c|c|c|c|c|c|}
\hline & Emotion & \multicolumn{7}{|c|}{ Gate condition (\# syllables) } \\
\hline & Disgust & 481 & 748 & 984 & 1290 & 1555 & 1958 & 2153 \\
\hline & Fear & 329 & 498 & 636 & 795 & 930 & 1151 & 1269 \\
\hline & Sadness & 405 & 626 & 815 & 1071 & 1286 & 1645 & 1846 \\
\hline
\end{tabular}

Pseudo-utterances were always gated at syllable boundaries from the offset of the utterance in gates of increasing syllable duration. 
full utterance was presented were labeled as "errors" and omitted from the calculation of emotion identification times (a total of 4381 data points were included). Mean emotion identification times were submitted to a one-way ANOVA with repeated measures on emotion (anger, disgust, fear, happiness, sadness, neutral).

Since the stimuli, procedures, and analyses adopted here were virtually identical to those of Pell and Kotz (2011), our experiment allows unprecedented comparisons of how recognition of emotional prosody evolves over time as a function of the gating direction, shedding light on how the position of acoustic patterns for detecting emotions influences recognition processes. For each of our three dependent measures (accuracy scores, confidence ratings, emotion identification times), we therefore performed a second analysis to directly compare the current results to those of Pell and Kotz (2011) by entering the between-groups factor of Presentation Direction (gating from offset vs. onset). Separate $t$-tests first compared the age and education (in years) of the current participant group $(n=40)$ with participants studied by Pell and Kotz (2011, $n=48)$; there was no difference in the formal education of the two samples [ 17 vs. 16 years, respectively; $\left.t_{(86)}=1.548 ; p=0.125\right]$, although participants in the present study were older on average [25 vs. 22 years; $t_{(86)}=2.578 ; p=$ $0.012]$. Given the age difference, we entered age as a covariate in separate mixed ANCOVAs on the Hu-scores, confidence ratings, and emotion identification times as described above with the additional grouping variable of presentation Direction (onset, offset) of key theoretical interest in these analyses. For all statistical analyses, a significance level of 5\% (two-sided) was selected and post-hoc comparisons (Tukey's HSD, $p<0.05$ ) were applied whenever a significant main or interactive effect was observed.

\section{RESULTS \\ ACCURACY (HU-SCORES) AND CONFIDENCE RATINGS Effects of backwards gating on accuracy and confidence scores}

Table 2 shows the mean accuracy of participants (\% correct target recognition) in each emotion and gate condition when utterances were presented from their offset, prior to correcting these scores for participant response bias. A 7 (Gate) $\times 6$ (Emotion) ANOVA performed on the unbiased emotion recognition rates (i.e., calculated $\mathrm{Hu}$-Scores) revealed a main effect of Gate duration $\left[F_{(6,228)}=390.48 ; p<0.001\right]$, Emotion $\left[F_{(5,190)}=\right.$
142.57; $p<0.001]$, and a significant interaction of these factors $\left[F_{(30,1140)}=10.684 ; p<0.001\right]$. Post hoc (Tukey's) tests of the interaction first considered how the recognition of each emotion evolved as a function of gate duration when sentences were gated from their offset. As shown in Figure 1, the recognition of fear, anger, and sadness significantly improved over the course of hearing the first three gates (i.e., the last three syllables of the utterance, $p s<0.003$ ) with no further accuracy gains by the fourth gate condition (Gate_4-7, ps > 0.115). In contrast, accurate recognition of neutral, happiness, and disgust each significantly improved over a longer time frame corresponding to the first four gate conditions (Gate_7 to Gate_4-7, ps <0.001) without further changes after this point $(p s>0.087)$.

Further inspection of the interaction then looked at emotional differences on accuracy at each gate condition. When listeners heard only the utterance-final syllable (Gate_7), fear and anger prosody were recognized significantly better than all other emotional voices ( $p s<0.006)$, and fear was also recognized significantly better than anger ( $p<0.001)$. After fear and anger, sad expressions were identified significantly better from the last syllable than happy and neutral expressions ( $p$ s $<0.001)$, which did not differ $(p=1.000)$, followed by disgust which was recognized more poorly than any other emotion $(p s<0.046)$. This pattern was similar for stimuli composed of the last two and the last three syllables (Gate_6-7 and Gate_5-7, respectively) but changed somewhat as stimulus duration increased. After presenting the last four syllables (Gate_4-7), fear continued to exhibit the highest accuracy score (this was true in all gate conditions; ps $<0.017$ ) but recognition of anger and sad expressions was equivalent $(p=1.0)$, followed by happiness which was recognized significantly better than disgust $(p<0.001)$. After the last five syllables were presented (Gate_3-7), angry, sad and happy sentences were recognized at a similar rate $(p s>0.555)$, surpassing neutral and disgust ( $p s<0.001$ ). In the two longest gate conditions (Gate_2-7, Gate_1-7), accuracy scores for anger, sad, happy and neutral sentences were not statistically different ( $p s>0.407$ ) while vocal expressions of fear and disgust were respectively the best and worst recognized from speech prosody ( $p$ s $<0.017)$.

The analysis of associated confidence ratings (on a scale of 1-7) was restricted to trials in which the emotional target of the prosody was correctly identified. Two male participants

Table 2 | Mean accuracy (\% target recognition) of the 40 listeners who judged pseudo-utterances conveying each emotion according to the gate duration, when utterances were gated from the offset of the sentence.

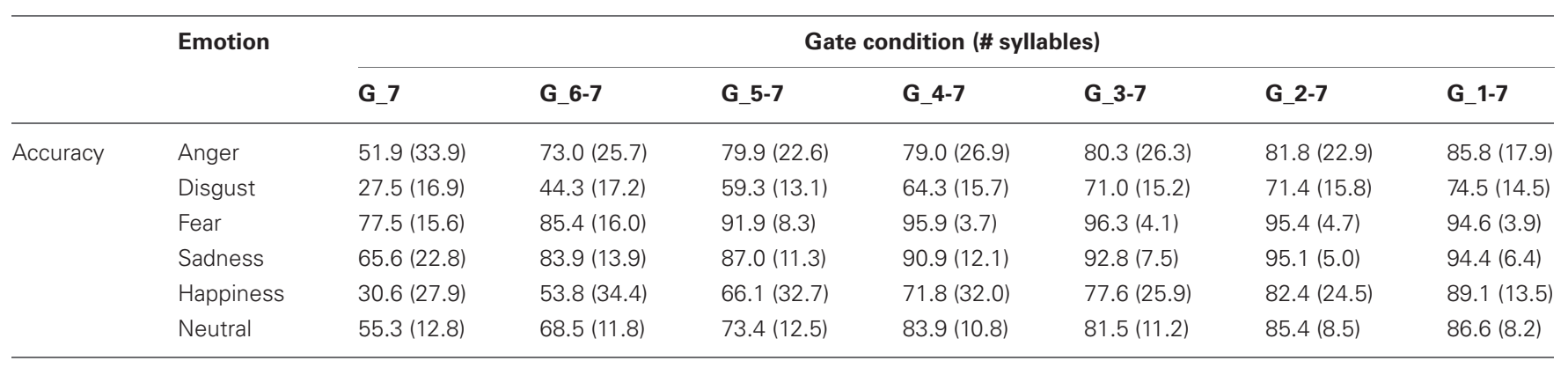

Standard deviations are shown in parentheses. 


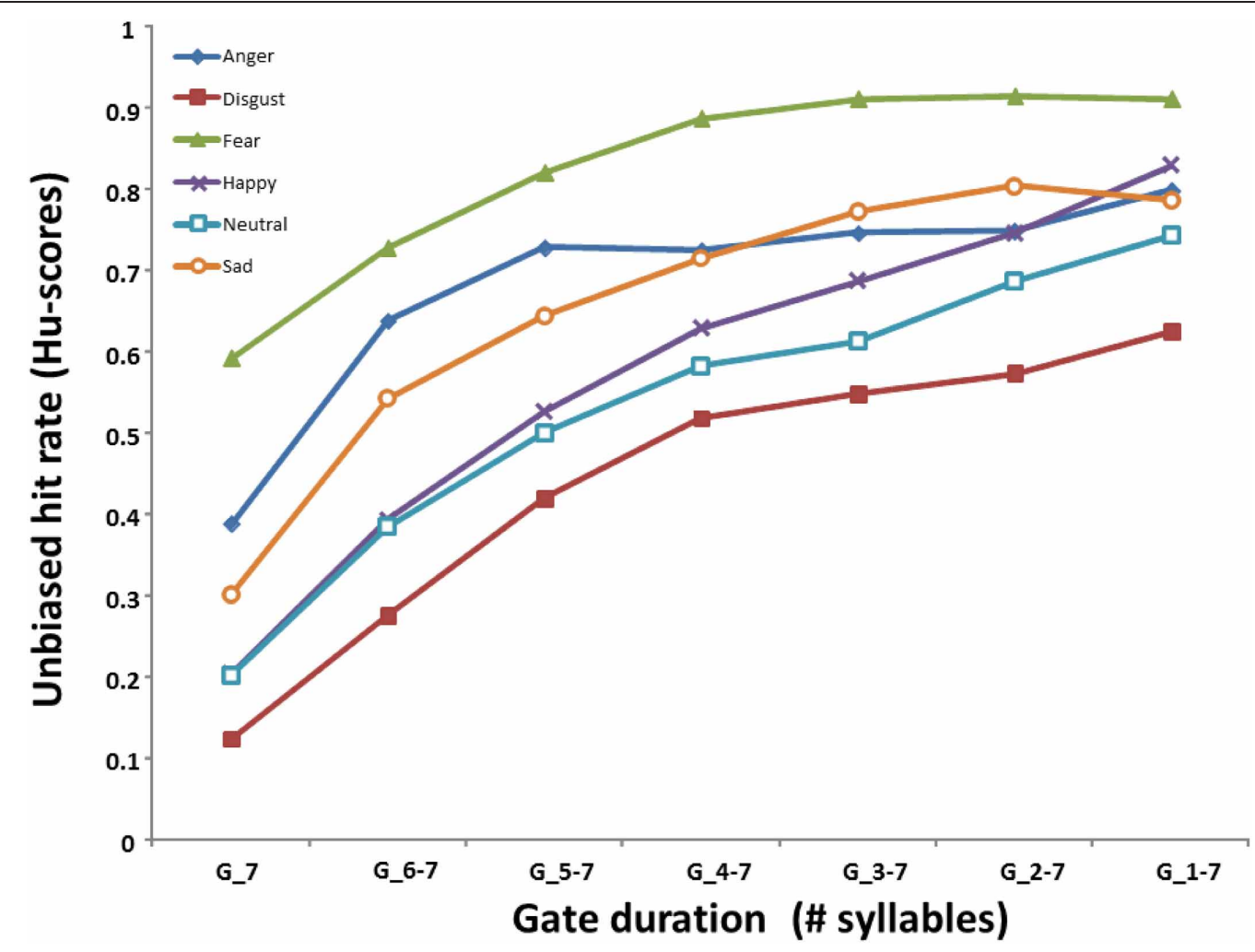

FIGURE 1 | Mean Hu-scores (unbiased accuracy) for each emotion as a function of the gate duration (number of syllables).

who failed to recognize any of the disgust expressions (producing an empty cell) were excluded from this analysis. The ANOVA on the confidence scores revealed a main effect of gate duration $\left[F_{(6,192)}=48.653 ; p<0.001\right]$, a main effect of emotional prosody $\left[F_{(5,160)}=46.991 ; p<0.001\right]$ and a significant interaction of Gate $\times$ Emotion $\left[F_{(30,960)}=3.814 ; p<0.001\right]$. Confidence scores tended to increase with stimulus/gate duration, although there were differences across emotions as a function of gate duration. After listening to the final one or two syllables, participants were significantly more confident about their detection of fear and anger ( $p s<0.001$ ) and least confident when they correctly recognized neutral and disgust ( $p s<0.001)$. Confidence ratings for happiness and sadness were between those extremes, differing significantly from the other two emotion sets $(p s<$ 0.048 ). By the third gate condition (Gate_5-7), confidence about neutral prosody began to increase over disgust $(p<0.001)$, and by the fourth gate condition and when exposed to longer stimuli, confidence ratings for fear, anger, happiness, and sadness were all comparable, although confidence about disgust remained significantly lower even when full utterances were presented (Gate_1-7).

\section{Impact of gating direction on accuracy and confidence scores}

The $2 \times 7 \times 6$ ANCOVA on Hu-scores gathered here and by Pell and Kotz (2011) showed a significant three-way interaction of Direction, Gate duration, and Emotion $\left[F_{(30,2550)}=12.636\right.$; $p<0.001]$. This interaction allowed us to explore the influence of presentation direction (onset vs. offset) on the accuracy of emotional prosody recognition as additional syllables revealed acoustic evidence about each emotion; these relationships are demonstrated for each emotion in Figure 2. Step-down analyses $(2 \times 7$ ANOVAs) showed that the interaction of Direction $\times$ Gate duration was significant for anger $\left[F_{(6,516)}=14.218 ; p<0.001\right]$, fear $\left[F_{(6,516)}=33.096 ; p<0.001\right]$, disgust $\left[F_{(6,516)}=10.851\right.$; $p<0.001]$, sadness $\left[F_{(6,516)}=11.846 ; p<0.001\right]$, and happiness $\left[F_{(6,516)}=9.663 ; p<0.001\right]$. For each of these emotions, recognition always improved when the end of utterances were heard first (i.e., when gated from their offset vs. onset), although the temporal region where accuracy improved within the utterance varied by emotion type. Post-hoc comparisons showed that anger and fear were recognized significantly better in the offset presentation condition even when little acoustic evidence was available; listeners detected anger better over the course of the first to third syllable in the offset vs. onset condition, and over the course of the first to sixth syllables for fear ( $p s<0.001$ ). Happiness showed an advantage in the offset condition beginning at the second up to the fourth gate $(p s=0.027)$, disgust showed a similar advantage beginning at the third to the fifth gate $(p<0.049)$, and sadness displayed the offset advantage beginning at the third up to the sixth gate $(p s<0.031)$. Interestingly, there was no effect of the direction of utterance presentation on the recognition of neutral prosody $\left[F_{(6,516)}=0.409 ; p=0.873\right]$.

The ANCOVA on confidence ratings between studies yielded a significant three-way interaction of Direction, Gate duration and Emotion $\left[F_{(30,2370)}=4.337 ; p<0.001\right]$. Step-down 

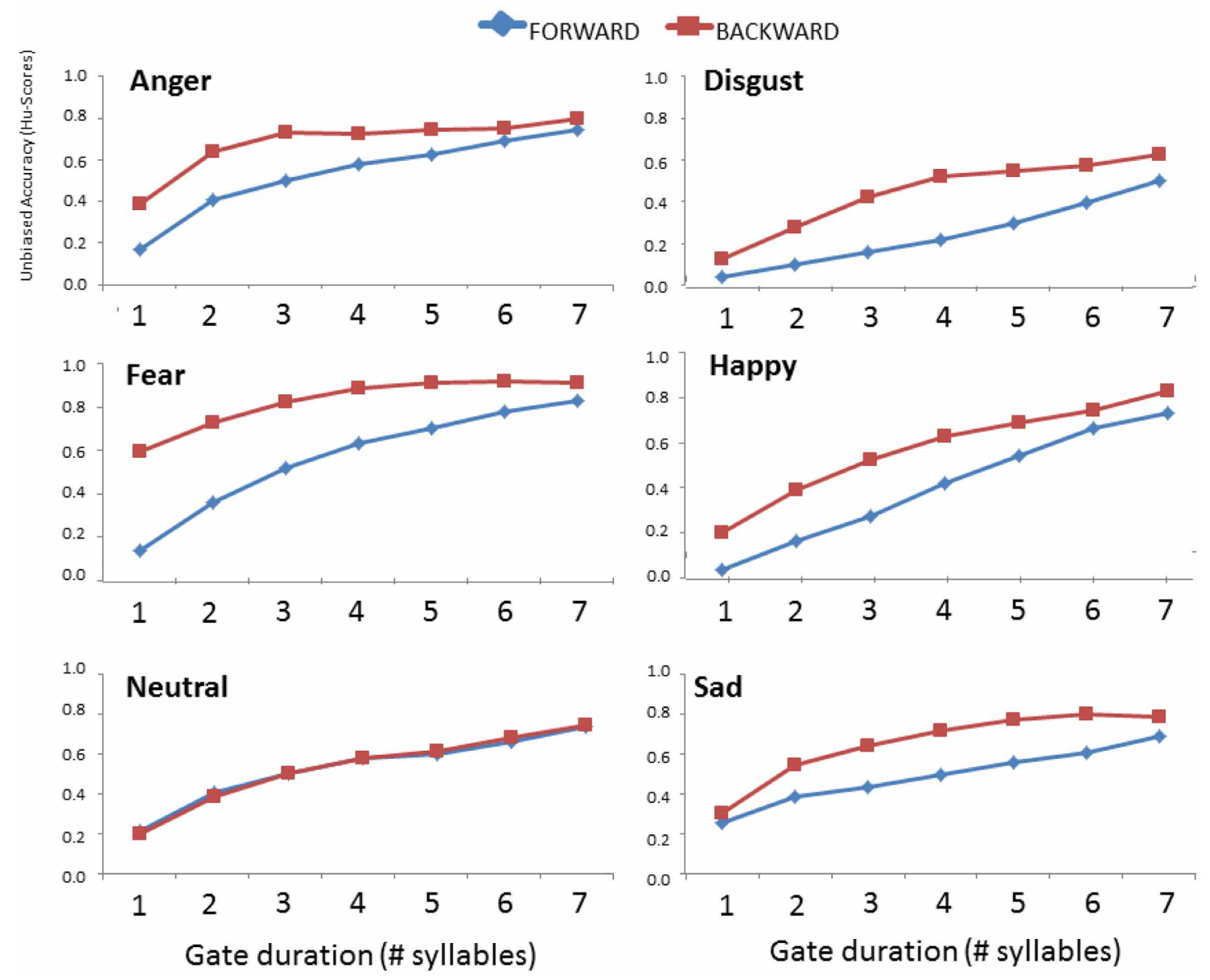

FIGURE 2 | Comparison of mean accuracy (Hu) scores for each emotion as a function of gate duration (number of syllables) and the direction of presentation (forward vs. backward). Data in the forward condition are taken from Pell and Kotz (2011).

analyses $(2 \times 7$ ANOVAs $)$ run separately by emotion showed that the interaction of Direction $\times$ Gate duration was significant for anger $\left[F_{(6,516)}=35.800 ; p<0.001\right]$, fear $\left[F_{(6,516)}=19.656\right.$; $p<0.001]$, happiness $\left[F_{(6,504)}=18.783 ; p<0.001\right]$, and sadness $\left[F_{(6,516)}=10.898 ; p<0.001\right]$. Listeners were more confident that they had correctly identified these four emotions only when one syllable was presented in isolation (i.e., at the first gate duration, $p s<0.049$ ), with increased confidence when they heard the sentence-final as opposed to the sentence-initial syllable. For disgust and neutral, the two-way interaction was also significant $\left[F_{(6,492)}=7.522 ; p<0.001 ; F_{(6,516)}=7.618 ; p<0.001\right.$, respectively] but post hoc tests revealed only minor differences in the pattern of confidence ratings in each presentation condition with no differences in listener confidence at specific gates ( $p$ s $>0.618$ ). These patterns are illustrated for each emotion in Figure 3.

\section{EMOTION IDENTIFICATION TIMES}

Effects of backwards gating on the time course of vocal emotion recognition

As described earlier, emotion identification times were computed by identifying the gate condition from sentence offset where the target emotion was correctly recognized for each item and participant, which was then converted into the precise time value of the gated syllables in milliseconds. A one-way ANOVA performed on the mean emotion identification times with repeated measures of emotion type (anger, disgust, fear, happiness, sadness and neutral) revealed a highly significant effect of emotion $\left[F_{(5,190)}=113.68\right.$; $p<0.001$ ]. As can be seen in Figure 3, fearful voices were correctly identified at the shortest presentation times $(M=427 \mathrm{~ms})$, significantly faster than sadness $(M=612 \mathrm{~ms})$, neutral $(M=$ $654 \mathrm{~ms})$ and anger $(M=672 \mathrm{~ms})$ which did not significantly differ one from another. These emotions required significantly less time to identify than happiness $(M=811 \mathrm{~ms})$, which in turn took significantly less time than disgust $(M=1197 \mathrm{~ms})$ which required the longest stimulus exposure for accurate recognition (all ps $<0.001$ ).

\section{Impact of gating direction on emotion identification times}

Finally, a $2 \times 6$ (Direction $\times$ Emotion) mixed ANCOVA was performed on the emotion identification times to compare the present results to those of Pell and Kotz (2011); this analysis revealed a significant interaction of presentation Direction and Emotion $\left[F_{(5,425)}=13.235 ; p<0.001\right]$ as also shown in 

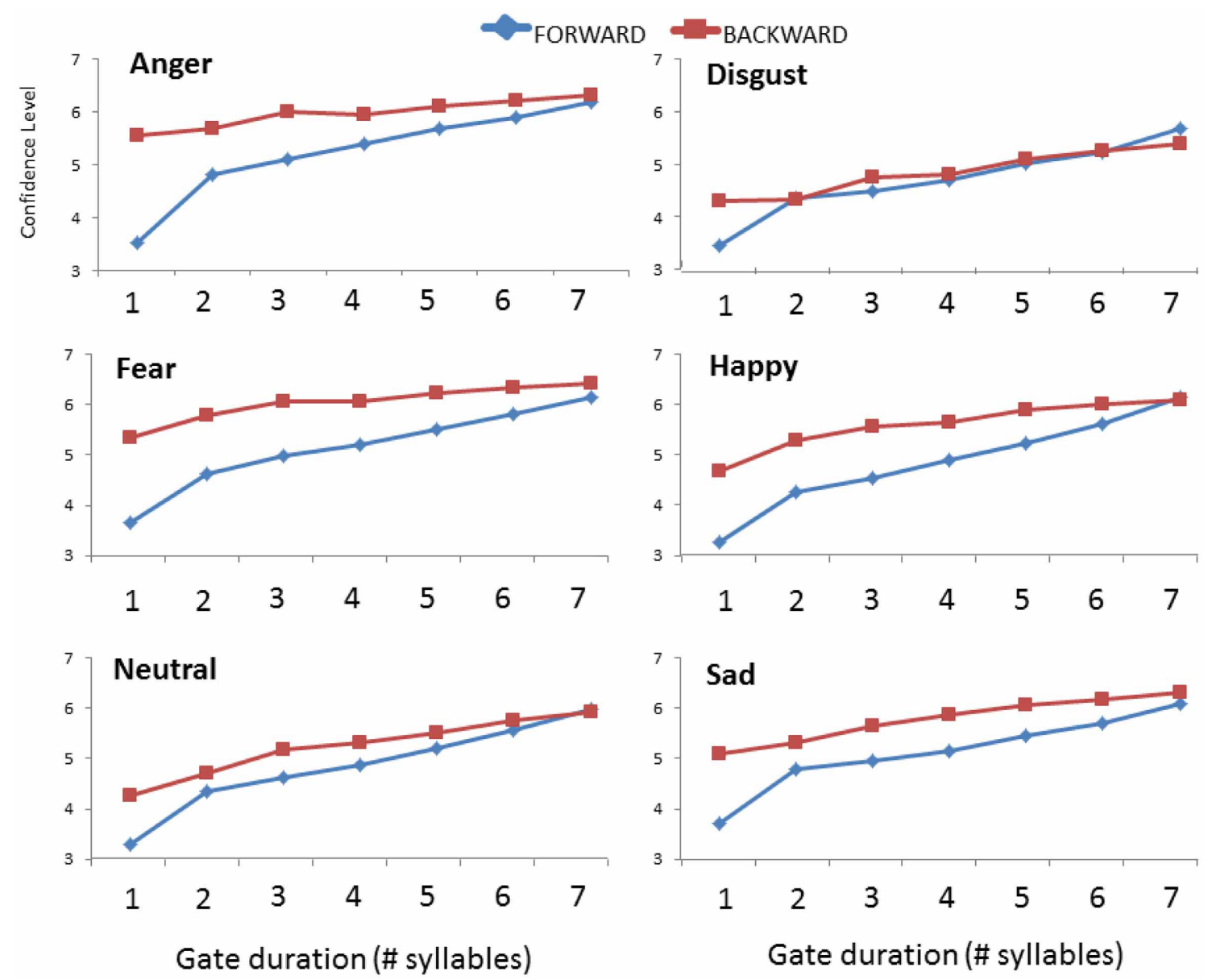

FIGURE 3 | Comparison of mean confidence ratings for each emotion as a function of gate duration (number of syllables) and the direction of presentation (forward vs. backward). Data in the forward condition are taken from Pell and Kotz (2011).

Figure 4 . The average time listeners required to correctly identify emotional prosody was significantly reduced when syllables were presented from the offset vs. onset of utterances, but only for disgust $(p<0.001)$ and happiness and $(p=0.050)$. In contrast to accuracy and confidence ratings, the manner in which utterances were gated had no significant impact on the amount of time listeners needed to recognize fear, sadness, anger, or neutral prosody (all ps $>0.157$ ).

\section{DISCUSSION}

Following recent work (Cornew et al., 2010; Pell and Kotz, 2011), this experiment sought a clearer understanding of how vocal expressions of basic emotion reveal their meanings in speech using a modified version of the gating paradigm, where emotionally-inflected pseudo-utterances were truncated and presented in excerpts of increasing syllable duration from the end of an utterance. While the current manner for presenting our stimuli might bear no immediate resemblance to how emotional speech is encountered in structured conversations-especially because our stimuli were only auditory and not spontaneously produced (see Barkhuysen et al., 2010 for a discussion on this topic)—our performance measures may help to understand some processes involved when listeners "walk in" on an emotional conversation, or have their attention directed to emotional speech in the environment that is already in progress, an experience that is common to everyday life. Critically, our design allowed important hypotheses to be tested concerning the evolution and associated time course of emotional prosody recognition (in English) as listeners are progressively exposed to representative acoustic cue configurations. In line with past findings, we found that listeners tended to be most accurate at recognizing vocal expressions of fear (Levitt, 1964; Zuckerman et al., 1975; Paulmann and Pell, 2011; Pell and Kotz, 2011) and least accurate for disgust (e.g., Scherer et al., 1991; Banse and Scherer, 1996) irrespective of how many syllables/gates were presented. Expressions of fear were also recognized from the shortest stimulus duration, implying that listeners need minimal input to recognize this emotion in speech (Pell and Kotz, 2011). Interestingly, emotion identification times were significantly reduced for certain emotions (happiness, disgust) when sentences were presented from their offset rather than their onset, and there were other apparent "advantages" to recognizing emotion prosody when listeners were first exposed to 


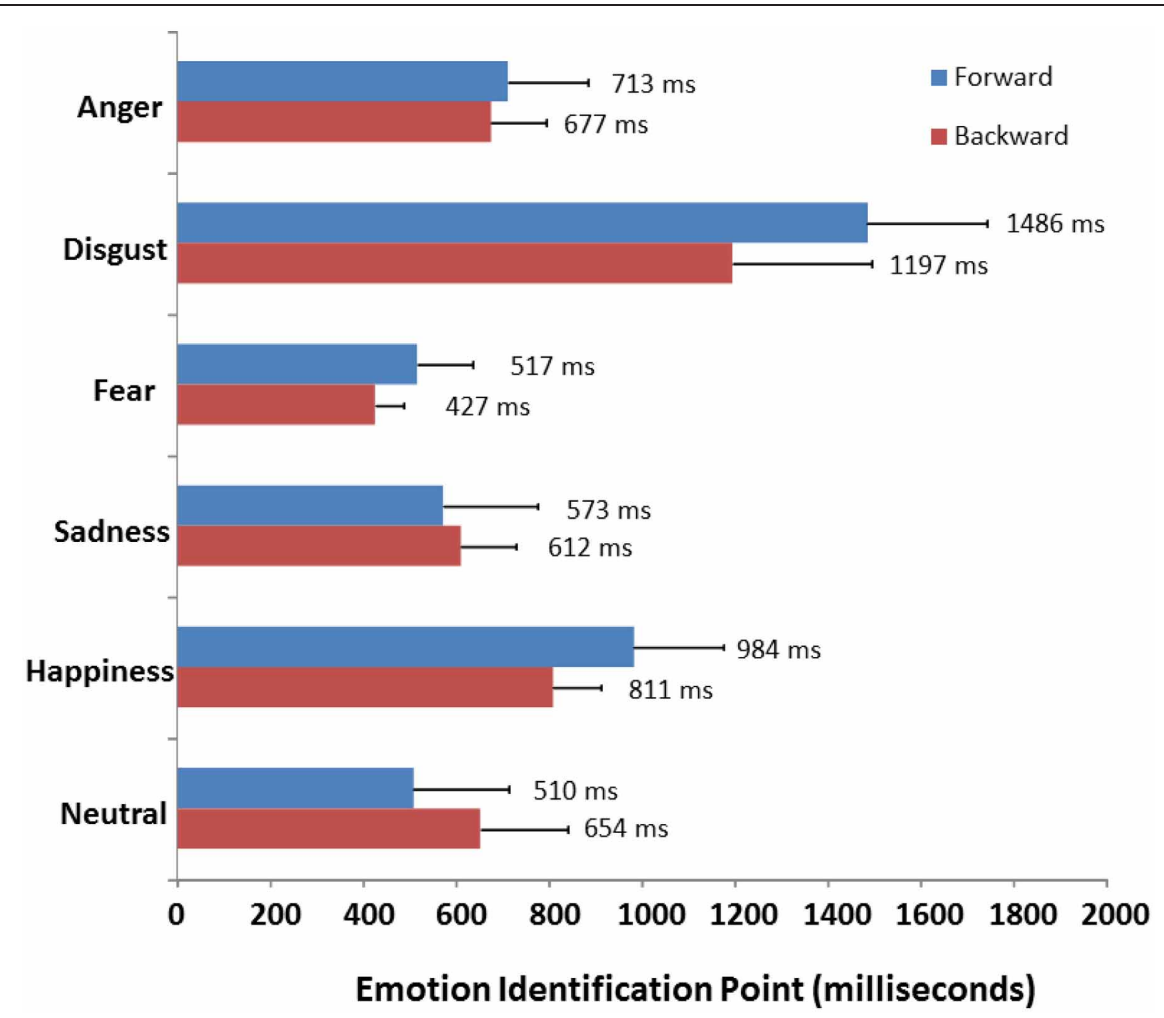

FIGURE 4 | Comparison of mean identification points (in milliseconds) for each emotion as a function of direction of presentation (forward vs. backward). Data in the forward condition are taken from Pell and Kotz (2011).

the end of utterances. These effects and their implications are discussed in detail below.

\section{EFFECTS OF GATING DIRECTION AND CUE LOCATION ON VOCAL EMOTION RECOGNITION}

Our data show that recognition of vocal emotions generally improves with the number of syllables presented, even when listeners hear utterance fragments in reverse order, but reaches a plateau for all emotions after hearing the last three to four syllables of the utterance. When viewed broadly, these findings suggest that "prototypical" acoustic properties for accessing knowledge about basic emotions from speech (Laukka, 2005; Pell et al., 2009) are decoded and consciously recognized at peak accuracy levels after processing three to four spoken syllables - approximating a mean stimulus duration of 600-1200 ms, depending on the emotion in question (review Table 1). This broad conclusion fits with observations of two previous gating studies that gated emotional utterances in syllabic units (Pell and Kotz, 2011) or in $250 \mathrm{~ms}$ increments (Cornew et al., 2010). However, there were notable emotion-specific recognition patterns as a function of gate duration; when stimuli were very short (i.e., only the final one or two syllables were presented) there was a marked advantage for detecting fear and anger when compared to the other expression types, and listeners were significantly more confident that they had correctly identified these two emotions based solely on the utterance-final syllable. As the gate duration gradually increased to five syllables (Gate_3-7), no further differences were observed in the ability to recognize anger, sadness, and happiness, although participants remained significantly more accurate for fear and significantly less accurate for disgust at all stimulus durations.

The observation that fear, and to a lesser extent anger, were highly salient to listeners at the end of utterances even when minimal acoustic information was present (i.e., the final syllable) is noteworthy. Leinonen and colleagues (1997) presented twosyllable emotional utterances in Finnish (the word [saara]) and reported higher recognition scores and distinct acoustic attributes of productions conveying fear and anger when compared to eight other emotional-motivational states, suggesting that these emotions are highly salient to listeners in acoustic stimuli of brief duration. Similarly, Pell and Kotz (2011) reported that recognition of most emotions improved over the full course of the utterance when they were gated from sentence onset and that certain emotions, such as happiness and fear, demonstrated clear gains in that study when listeners processed the last two syllables of the utterance. When combined with our current findings, this implies that syllables located towards the end of an utterance provide especially powerful cues for identifying basic emotions encoded in spoken language. This argument is supported by our direct statistical comparisons of the two data sets when utterances were gated from their onset vs. offset; we found that presentation direction had a significant impact on the accuracy and confidence levels of English listeners, with improved recognition of all emotions except neutral when participants heard utterances 
commencing with the last syllable. Gating utterances from their offset also reduced mean emotion identification times for some emotions (happiness, disgust) as elaborated below. In contrast, there was no evidence in our data that listeners were at an advantage to recognize emotional prosody when utterances were gated from their onset, with the possible exception of accuracy rates for sadness that were somewhat higher in the onset condition at very short gate intervals.

Why would natural, presumably biologically-specified codes for signaling emotions in the voice (e.g., Ekman, 1992; Wilson and Wharton, 2006) bear an important relationship to the temporal features of spoken language? This phenomenon, which has been highlighted at different times (Cosmides, 1983; Scherer, 1988), could be explained by the accent structure of utterances we presented for emotion recognition and by natural processes of speech production, factors which both contribute to the "socialization" or shaping of vocal emotion expressions in the context of spoken language. It is well known that the accent/phrase structure of speech, or the relative pattern of weak vs. strong syllables (or segments) in a language, can be altered when speakers experience and convey vocal emotions (Ladd, 1996). For example, speakers may increase or decrease the relative prominence of stressed syllables (through local changes in duration and pitch variation) and/or shift the location or frequency of syllables that are typically accented in a language, which may serve as an important perceptual correlate of vocal emotion expressions (Bolinger, 1972; Cosmides, 1983). Related to the notion of local prominence, there is a well-documented propensity for speakers to lengthen syllables located in word- or phrase-final position ("sentence-final lengthening," Oller, 1973; Pell, 2001), sometimes on the penultimate syllable of certain languages (Bolinger, 1978), and other evidence that speakers modulate their pitch in final positions to encode gradient acoustic cues that refer directly to their emotional state (Pell, 2001) to give to the final position of sentences a special impact in the identification of the emotional quality of the voice.

The observation here that cues located toward the end of an utterance facilitated accurate recognition of most emotions in English likely re-asserts the importance of accent structure during vocal emotion processing (Cosmides, 1983; Ladd et al., 1985). More specifically, it implies that sentence-final syllables in many languages could act as a vehicle for reinforcing the speaker's emotion state vis-à-vis the listener in an unambiguous and highly differentiated manner during discourse (especially for fear and anger). Inspection of the mean syllable durations of gated stimuli presented here and by Pell and Kotz (2011) confirm that while there were natural temporal variations across emotions, the duration of utterance-final syllables $(M=386 \mathrm{~ms}$, range $=$ 329-481) was more than double that of utterance-initial syllables $(M=165 \mathrm{~ms}$, range $=119-198)$, the latter of which were always unstressed in our study. In comparison, differences in the cumulative duration of gates composed of two syllables $(M=600$ vs. 516 in the offset vs. onset conditions, respectively) or three syllables $(M=779$ vs. 711$)$ were relatively modest between the two studies, and these stimulus durations were always composed of both weak and stressed syllables. This difference of duration observed is in line with the above described propensity of speakers to lengthen syllables located in the final position of the sentences. Also, given the structure of the pseudo-utterances (see Section Appendix), it should be noted that the forward presentation of pseudo-utterances might differ from the backward presentation in terms of expectations of the participants. In Pell and Kotz (2011), the first gate was always a pronoun or a determiner and was always followed by the first syllable of a pseudo-verb, whereas in the present experiment, the two first gates were always the two final syllables of a pseudo-word. It is difficult to know whether participants may have developed some expectations about the following syllable and to what extent these expectations could have impacted the identification of the prosody. We cannot exclude that these expectations could have been more difficult to make in the backward condition, when the gates were presented in a reverse order, altering how participants focused on the emotional prosody of the sentences. However, such an interpretation would not explain why the direction of presentation did not influence the performance of participants when sentences were uttered with a neutral note and why this influence was limited to some specific gates when the sentences were spoken in an emotional way.

Nevertheless, these results suggest that there is a certain alignment in how speakers realize acoustic targets that refer to semantically-dictated stress patterns and emotional meanings in speech, demonstrating that recognition of vocal emotional expressions is shaped to some extent by differences in the temporal (accent) structure of language and that emotional cues are probably not equally salient throughout the speech signal. Further studies that compare our findings with data from other languages will clearly be needed to advance specific hypotheses about how vocal emotion expressions may have become "domesticated" in the context of spoken language. For example, we could replicate forward and backward gating experiments in another stressed-language like German, and see if critical cues in the identification of some emotions could be located at different places of a sentence. We could also compare forward and backward presentation of pseudo-sentences in a language that does not use accentuated stress such as French, which supposedly would lead to similar results in the time needed to identify emotional prosody irrespective of the direction of presentation of the sentences.

\section{Further reflections on the time course of vocal emotion recognition}

While our data show that the position of emotionally meaningful cues plays a role in how vocal emotions are revealed to listeners, they simultaneously argue that the average time needed to accurately decode most basic emotions in speech is relatively constant irrespective of gating method (syllables vs. $250 \mathrm{~ms}$ increments) or stimulus set (Cornew et al., 2010; Pell and Kotz, 2011). When mean emotion identification times were computed here, fear required the least amount of stimulus exposure to recognize $(M=427 \mathrm{~ms})$, followed by sadness $(M=612 \mathrm{~ms})$, neutral $(M=654 \mathrm{~ms})$, anger $(M=677 \mathrm{~ms})$, happiness $(M=811 \mathrm{~ms})$, and disgust $(M=1197 \mathrm{~ms})$. With the exception of neutral which took slightly (although not significantly) longer to detect when utterances were gated in reverse, this emotion-specific pattern precisely mirrors the one reported by Pell and Kotz (2011) for the same six emotions and replicates Cornew et al.'s (2010) data 
for neutral, anger, and happy expressions when utterances were gated in $250 \mathrm{~ms}$ units. When the mean emotion identification times recorded here are compared to those reported by Pell and Kotz (2011) and Cornew et al. (2010), it can be said that recognition of fear occurs approximately in the range of 425-525 ms $(427,517 \mathrm{~ms})$, sadness in the range of $600 \mathrm{~ms}(612,576 \mathrm{~ms})$, anger in the range of $700 \mathrm{~ms}(677,710,723 \mathrm{~ms})$, happiness in the range of $800-900 \mathrm{~ms}(811,977,802 \mathrm{~ms})$, and disgust requires analysis of at least $1200 \mathrm{~ms}$ of speech $(1197,1486 \mathrm{~ms})$. As pointed out by Pell and Kotz (2011), the time needed to identify basic emotions from their underlying acoustic cues does not simply reflect characteristic differences in articulation rate across emotions (e.g., Banse and Scherer, 1996; Pell et al., 2009), since expressions of sadness are routinely slower and often twice the duration of comparable fear expressions, and yet these two emotions are accurately recognized from speech stimuli of the shortest duration. Rather, it can be claimed that prototypical cues for understanding vocal emotions are decoded and consciously retrievable over slightly different epochs in the 400-1200 ms time window, or after hearing roughly 2-4 syllables in speech. The idea that emotional meanings begin to be differentiated after hearing around $400 \mathrm{~ms}$ of speech fits with recent priming data using behavioral paradigms (Pell and Skorup, 2008) and event-related potentials (ERPs, Paulmann and Pell, 2010) as well as recent neuro-cognitive models on the time course and cognitive processing structure of vocal emotion processing (Schirmer and Kotz, 2006).

Evidence that vocal expressions of certain negative emotions, such as fear, sadness, or anger, require systematically less auditory input to decode accurately, whereas expressions of happiness and disgust take much longer, may be partly explained by the evolutionary prevalence and significance of negative emotions over positive emotions (e.g., Cacioppo and Gardner, 1999). Expressions that signal threat or loss must be decoded rapidly to avoid detrimental outcomes of great urgency to the organism; this negativity bias has been observed elsewhere in response to facial (Carretié et al., 2001) and vocal expressions of fear and anger (Calder et al., 2001, 2004), and would explain why fear prosody was recognized more accurately and faster than any other emotional expression in the voice (Levitt, 1964). The biological importance of rapidly differentiating negative vocal signals (e.g., Scherer, 1986) potentially explains why the amount of temporal acoustic information, and not the position of critical cues, appears to be the key factor governing the time course of recognizing fear, anger, and sadness, since we found no significant differences in emotion identification times for these emotions between our two studies.

In contrast, happy and disgust took significantly longer to identify and were the only emotions for which recognition times varied significantly as a function of gating direction (with a reduction in emotion recognition times of approximately $200 \mathrm{~ms}$ and $300 \mathrm{~ms}$ between studies, respectively). Difficulties recognizing disgust from prosody are well documented in the literature (Scherer, 1986; Scherer et al., 1991; Jaywant and Pell, 2012) and are sometimes attributed to the fact that disgust in the auditory modality is more typical in the form of affective bursts such as "yuck" or "eeeew" (Scherer, 1988; Simon-Thomas et al., 2009). It is possible that identifying disgust from running speech, as required here and by Pell and Kotz (2011), activates additional social meanings that take more time to analyze and infer than the decoding of pure biological signals such as fear, sadness, and anger. For example, it has been suggested that there are qualitatively different expressions of disgust in the visual (Rozin et al., 1994) and auditory (Calder et al., 2010) modality, including a variant related to violations of moral standards that is often conveyed in running speech, as opposed to physical/visceral expressions of disgust which are better conveyed through exclamations (yuck!). If presentation of disgust utterances engendered processes for inferring a speaker's social or moral attitude from vocal cues, a more symbolic function of prosody, one might expect a much slower time course as witnessed here. A similar argument may apply to our results for happiness; although this emotion is typically the quickest emotion to be recognized in the visual modality (Tracy and Robins, 2008; Palermo and Coltheart, 2004; Calvo and Nummenmaa, 2009), it exhibits a systematically slower time course in spoken language (Cornew et al., 2010; Pell and Kotz, 2011). Like disgust, happiness may also be communicated in a more rapid and reliable manner by other types of vocal cues that accompany speech, such as laughter (e.g., Szameitat et al., 2010). In addition, there is probably a need to differentiate between different types of vocal expressions of happiness which yield different rates of perceptual recognition (Sauter and Scott, 2007). Nonetheless, our results strongly imply that speakers use prosody to signal happiness, particularly towards the end of an utterance, as a conventionalized social cue directed to the listener for communicating this emotion state (Pell, 2001; Pell and Kotz, 2011), perhaps as a form of self-presentation and inter-personal expression of social affiliation. Further inquiry will be needed to test why disgust and happiness appear to be more socially mediated than other basic emotions, influencing the time course of their recognition in speech, and to define the contexts that produce variations in these expressions.

Interestingly, the recognition of neutral prosody was uniquely unaffected by the manner in which acoustic information was unveiled in the utterance, with no significant effects of presentation direction on accuracy, confidence ratings, or emotion identification times between studies. This tentatively suggests that the identification of neutrality, or a lack of emotionality in the voice, can be reliably inferred following a relatively standard amount of time in the range of 400-650 ms of stimulus exposure (Cornew et al., 2010; Pell and Kotz, 2011). Since our measures of recognition include conscious interpretative (naming) processes and are biased somewhat by the gating method, our data on the time course for neutral prosody are not inconsistent with results showing the on-line differentiation of neutrality/emotionality in the voice at around $200 \mathrm{~ms}$ after speech onset, as inferred from amplitude differences in the P200 ERP component when German utterances were presented to listeners (Paulmann et al., 2008). One can speculate that listeners use a heuristic or default process for recognizing neutral voices whenever on-line analysis of prosody does not uncover evidence of emotionally meaningful cue configurations; presumably, this process for rejecting the presence of known acoustic patterns referring to emotions, like the process for decoding known patterns, is accomplished over a relatively stable time interval. To test these possibilities, 
it would be interesting to modify neutral sentences by inserting local variations in emotionally-meaningful acoustic features at critical junctures in time to determine if this "resets the clock" for inferring the presence or absence of emotion in speech.

\section{CONCLUSION}

Following recent on-line (ERP) studies demonstrating that vocal emotions are distinguished from neutral voices after $200 \mathrm{~ms}$ of speech processing (Paulmann and Kotz, 2008), and that emotionspecific differences begin to be detected in the 200-400 ms time window (Alter et al., 2003; Paulmann and Pell, 2010), our data shed critical light on the time interval where different emotionspecific meanings of vocal expressions are fully recognized and available for conscious retrieval. While it seems likely that the phrase structure of language governs local opportunities for speakers to encode emotionally-meaningful cues that are highly salient to the listener, at least in certain contexts, there are remarkable consistencies in the amount of time listeners must monitor vocal cue configurations to decode emotional (particularly threatening) meanings. As such, the idea that there are systematic

\section{REFERENCES}

Alter, K., Rank, E., Kotz, S. A., Toepel, U., Besson, M., Schirmer, A., et al. (2003). Affective encoding in the speech signal and in event-related brain potentials. Speech Commun. 40, 61-70. doi: 10.1016/S0167-6393(02)00075-4

Banse, R., and Scherer, K. R. (1996). Acoustic profiles in vocal emotion expression. J. Pers. Soc. Psychol. 70, 614-636. doi: 10.1037/00223514.70.3.614

Barkhuysen, P., Krahmer, E., and Swerts, M. (2010). Crossmodal and incremental perception of audiovisual cues to emotional speech. Lang. Speech 53, 1-30. doi: 10.1177/0023830909348993

Becker, D. V., Neel, R., Srinivasan, N., Neufeld, S., Kumar, D., and Fouse, S. (2012). The vividness of happiness in dynamic facial displays of emotion. PLoS ONE 7:e26551. doi: 10.1371/journal.pone.0026551

Boersma, P., and Weenink, D. (2012). Praat: Doing Phonetics by Computer [Computer Program]. Version 5.3.21.

Bolinger, D. (1978). "Intonation across languages," in Universals of Human Language, vol. II. Phonology, ed J. Greenberg (Palo Alto, CA: Stanford University Press), 471-524.

Bolinger, D. (1972). Accent is predictable (if you're a mind reader). Language 48, 633-644. doi: 10.2307/412039

Cacioppo, J. T., and Gardner, W. L. (1999). Emotion. Аnnu. Rev. Psychol. 50, 191-214. doi: 10.1146/annurev.psych.50.1.191
Calder, A. J., Keane, J., Lawrence, A. D., and Manes, F. (2004). Impaired recognition of anger following damage to the ventral striatum. Brain 127: 1958-1969. doi: 10.1093/brain/awh214

Calder, A. J., Lawrence, A. D., and Young, A. W. (2001). Neuropsychology of fear and loathing. Nat. Rev. Neurosci. 2, 352-363. doi: 10.1038/35072584

Calder, A. J., Keane, J., Young, A. W., Lawrence, A. D., Mason, S., and Barker, R. (2010). The relation between anger and different forms of disgust: implications for emotion recognition impairments in Huntington's disease. Neuropsychologia 48, 2719-2729. doi: $\quad 10.1016 /$ j.neuropsychologia. 2010.05.019

Calvo, M. G., and Nummenmaa, L. (2009). Eye-movement assessment of the time course in facial expression recognition: neurophysiological implications. Cogn. Affect. Behav. Neurosci. 9, 398-411. doi: 10.3758/CABN.9.4.398

Carretié, L., Mercado, F., Tapia, M., and Hinojosa, J. A. (2001). Emotion, attention, and the "negativity bias", studied through event-related potentials. Int. J. Psychophysiol. 41, 75-85. doi: 10.1016/S0167-8760(00)00195-1

Cornew, L., Carver, L., and Love, T. (2010). There's more to emotion than meets the eye: a processing bias for neutral content in the domain of emotional prosody. Cogn. Emot. 24: 1133-1152. doi: $10.1080 / 02699930903247492$

differences in the time course for arriving at vocal emotional meanings is confirmed. To gather further information on how social factors influence the communication of vocal emotional meanings, future studies using the gating paradigm could present emotional utterances to listeners in their native vs. a foreign language; this could reveal whether specificities in the time course for recognizing emotions manifest in a similar way for native speakers of different languages, while testing the hypothesis that accurate decoding of vocal emotions in a foreign language is systematically delayed due to interference at the phonological level (Van Bezooijen et al., 1983; Pell and Skorup, 2008).

\section{ACKNOWLEDGMENTS}

This research was financially supported by a Discovery Grant from the Natural Sciences and Engineering Research Council of Canada (RGPIN 203708-11 to Marc D. Pell). Assistance from the McGill University Faculty of Medicine (McLaughlin Postdoctoral Fellowship to Simon Rigoulot) and the KonradAdenauer-Foundation (to Eugen Wassiliwizky) are also gratefully acknowledged.

Cosmides, L. (1983). Invariances in the acoustic expression of emotion during speech. J. Exp. Psychol. Hum. Percept. Perform. 9 (6), 864-881. doi: 10.1037/0096-1523.9.6.864

Ekman, P. (1972). "Universal and cultural differences in facial expressions of emotions," in Nebraska Symposium on Motivation, 1971, ed J. K. Cole (Lincoln: University of Nebraska Press), 207-283.

Ekman, P. (1992). An argument for basic emotions. Cogn. Emot. 6, 169-200. doi: $10.1080 / 02699939208411068$

Ekman, P., Levenson, R. W., and Friesen, W. V. (1983). Autonomic nervous system activity distinguishes between emotions. Science 221, 1208-1210. doi: $10.1126 /$ science.6612338

Grosjean, F. (1980). Spoken word recognition processes and the gating paradigm. Percept. Psychophys. 18, 267-283. doi: 10.3758/BF03204386

Grosjean, F. (1996) Gating. Lang. Cogn. Process. 11, 597-604. doi: 10.1080/016909696386999

Hess, U., Beaupré, M. G., and Cheung, N. (2002). "Who to whom and why - cultural differences and similarities in the function of smiles," in An Empirical Reflection on the Smile, eds M. Abel and C. H. Ceia (Lewiston, NY: The Edwin Mellen Press), 187-216.

Izard, C. E. (1971). The Face of Emotion. New York, NY: Appleton-CenturyCrofts.

Jaywant, A., and Pell, M. D. (2012). Categorical processing of negative emotions from speech prosody.
Speech Commun. 54, 1-10. doi: 10.1016/j.specom.2011.05.011

Johnstone, T., and Scherer, K. R. (2000). "Vocal communication of emotion," in Handbook of Emotions, 2nd Edn. eds M. Lewis and J. Haviland (New York, NY: Guilford Press), 220-235.

Juslin, P. N., and Laukka, P. (2003). Communication of emotions in vocal expression and music performance: different channels, same code? Psychol. Bull. 129, 770-814. doi: 10.1037/0033-2909.129.5.770

Ladd, D. R. (1996). Intonational Phonology. Cambridge, UK: Cambridge University Press.

Ladd, D. R., Silverman, K., Tolkmitt, F., Bergmann, G., and Scherer, K. R. (1985). Evidence for the independent function of intonation contour type, voice quality and F0 range in signaling speaker affect. J. Acoust. Soc. Am. 78, 435-444. doi: 10.1121/1.392466

Laukka, P. (2005). Categorical perception of vocal emotion expressions. Emotion 5, 277-295. doi: 10.1037/1528-3542.5.3.277

Laukka, P., Juslin, P., and Bresin, R. (2005). A dimensional approach to vocal expression of emotion. Cogn. Emot. 19, 633-653. doi: 10.1080/02699930441000445

Leinonen, L., Hiltunen, T., Linnankoski, I., and Laakso, M.-L. (1997). Expression of emotionalmotivational connotations with a one-word utterance. J. Acoust. Soc. Am. 102, 1853-1863. doi: $10.1121 / 1.420109$

Levenson, R. W. (1992). Autonomic nervous system differences among 
emotions. Psychol. Sci. 3, 23-27. doi: $\quad$ 10.1111/j.1467-9280.1992. tb00251.x

Levitt, E. A. (1964). "The relationship between abilities to express emotional meanings vocally and facially," in The Communication of Emotional Meaning, ed J. R. Davitz (New York, NY: McGrawHill), 87-100.

Oller, D. K. (1973). The effect of position in utterance on speech segment duration in English. J. Acoust. Soci. Am. 54, 1235-1247. doi: 10.1121/1.1914393

Palermo, R., and Coltheart, M. (2004). Photographs of facial expression: accuracy, response times, and ratings of intensity. Behav. Res. Meth. Instrum. Comput. 36, 634-638. doi: 10.3758/BF03206544

Paulmann, S., and Kotz, S. A. (2008). Early emotional prosody perception based on different speaker voices. Neuroreport, 19, 209-213. doi: 10.1097/WNR.0b013e3282f454db

Paulmann, S., and Pell, M. D. (2010). Contextual influences of emotional speech prosody on face processing: how much is enough? Cogn., Affect. Behav. Neurosci. 10, 230-242. doi: 10.3758/CABN.10.2.230

Paulmann, S., and Pell, M. D. (2011). Is there an advantage for recognizing multi-modal emotional stimuli? Mot. Emot. 35, 192-201. doi: 10.1007/s11031-011-9206-0

Paulmann, S., Pell, M. D., and Kotz, S. A. (2008). Functional contributions of the basal ganglia to emotional prosody: evidence from ERPs. Brain Res. 1217, 171-178. doi: 10.1016/j.brainres.2008.04.032

Pell, M. D. (2001). Influence of emotion and focus location on prosody in matched statements and questions. J. Acoust. Soc. Am. 109, 1668-1680. doi: 10.1121/1.1352088

Pell, M. D., and Baum, S. R. (1997). Unilateral brain damage, prosodic comprehension deficits, and the acoustic cues to prosody. Brain Lang. 57, 195-214. doi: 10.1006/brln.1997.1736
Pell, M. D., and Kotz, S. (2011). On the time course of vocal emotion recognition. PLOS ONE 6:e27256. doi: 10.1371/journal.pone.0027256

Pell, M. D., Paulmann, S., Dara, C., Alasseri, A., and Kotz, S. A. (2009). Factors in the recognition of vocally expressed emotions: a comparison of four languages. J. Phonet. 37, 417-435. doi: 10.1016/j.wocn.2009. 07.005

Pell, M. D., and Skorup, V. (2008). Implicit processing of emotional prosody in a foreign versus native language. Speech Commun. 50, 519-530. doi: 10.1016/j.specom. 2008.03.006

Rigoulot, S., and Pell, M. D. (2012). Seeing emotion with your ears: emotional prosody implicitly guides visual attention to faces. PLOS ONE 7:e30740. doi: 10.1371/journal.pone.0030740

Rozin, P., Lowery, L., and Ebert, R. (1994). Varieties of disgust faces and the structure of disgust. J. Pers. Soc. Psychol. 66, 870-881. doi: 10.1037/0022-3514.66.5.870

Sauter, D., Eisner, F., Ekman, P., and Scott, S. K. (2010). Cross-cultural recognition of basic emotions through nonverbal emotional vocalizations. Proc. Natl. Acad. Sci. U.S.A. 107, 2408-2412. doi: 10.1073/pnas.0908239106

Sauter, D., and Scott, S. K. (2007). More than one kind of happiness: can we recognize vocal expressions of different positive states? Mot. Emot. 31, 192-199. doi: 10.1007/s11031-0079065-x

Scherer, K. R. (1986) Vocal affect expression: a review and a model for future research. Psychol. Bull. 99, 143-165. doi: 10.1037/0033 2909.99.2.143

Scherer, K. R. (1988). On the symbolic functions of vocal affect expression. J. Lang. Soc. Psychol. 7, 79-100. doi: $\quad 10.1177 / 0261927 X 8800$ 700201

Scherer, K. R. (2009). Emotions are emergent processes: they require a dynamic computational architecture. Philos. Trans. $R$. Soc. B 364, 3459-3474. doi: 10.1098/rstb.2009.0141

Scherer, K. R., Banse, R., and Wallbott, H. G. (2001) Emotion inferences from vocal expression correlate across language and cultures. J. Cross Cult. Psychol. 32, 76-92. doi: 10.1177/0022022101032001009

Scherer, K. R., Banse, R., Wallbott, H. G., and Goldbeck, T. (1991) Vocal cues in emotion encoding and decoding. Mot. Emot. 15, 123-148. doi: 10.1007/BF00995674

Schirmer, A., and Kotz, S. A. (2006). Beyond the right hemisphere: brain mechanisms mediating vocal emotional processing. Trends Cogn. Sci. 10, 24-30. doi: 10.1016/j.tics.2005.11.009

Simon-Thomas, E., Keltner, D. Sauter, D., Sinicropi-Yao, L., and Abramson, A. (2009) The voice conveys specific emotions: evidence from vocal burst displays. Emotion 9, 838-846. doi: 10.1037/ a0017810

Sobin, C., and Alpert, M. (1999). Emotions in speech: the acoustic attributes of fear, anger, sadness and joy. J. Psycholinguist. Res. 28, 347-365. doi: 10.1023/A:1023237014909

Szameitat, D. P., Kreifelts, B., Alter, K., Szameitat, A. J., Sterr, A., Grodd, W., et al. (2010). It is not always tickling: distinct cerebral responses during perception of different laughter types. Neuroimage 53, 1264-1271. doi: 10.1016/j.neuroimage.2010. 06.028

Thompson, W. F., and Balkwill, L.-L. (2006). Decoding speech prosody in five languages. Semiotica 158, 407-24.

Tracy, J. L., and Robins, R. W. (2008). The automaticity of emotion recognition. Emotion 8, 81-95. doi: 10.1037/1528-3542.8.1.81

Van Bezooijen, R., Otto, S., and Heenan, T. (1983). Recognition of vocal expressions of emotion: a three-nation study to identify universal characteristics. J. Cross
Cult. Psychol. 14, 387-406. doi: 10.1177/0022002183014004001

Wagner, H. L. (1993) On measuring performance in category judgment studies of nonverbal behavior. J. Nonverb. Behav. 17, 3-28. doi: 10.1007/BF00987006

Wallbott, H. G., and Scherer, K. R. (1986). Cues and channels in emotion recognition. J. Personal. Soc. Psychol. 51, 690-99. doi: 10.1037/0022-3514.51.4.690

Wilson, D., and Wharton, T. (2006). Relevance and prosody. J. Pragmat. 38, 1559-1579. doi: 10.1016/j.pragma.2005.04.012

Zuckerman, M., Lipets, M. S., Koivumaki, J. H., and Rosenthal, R. (1975). Encoding and decoding nonverbal cues of emotion. J. Pers. Soc. Psychol. 32, 1068-1076. doi: 10.1037/0022-3514.32.6.1068

Conflict of Interest Statement: The authors declare that the research was conducted in the absence of any commercial or financial relationships that could be construed as a potential conflict of interest.

Received: 22 February 2013; paper pending published: 27 March 2013; accepted: 04 June 2013; published online: 24 June 2013.

Citation: Rigoulot S, Wassiliwizky E and Pell MD (2013) Feeling backwards? How temporal order in speech affects the time course of vocal emotion recognition. Front. Psychol. 4:367. doi: 10.3389/fpsyg. 2013.00367

This article was submitted to Frontiers in Emotion Science, a specialty of Frontiers in Psychology.

Copyright (c) 2013 Rigoulot, Wassiliwizky and Pell. This is an open-access article distributed under the terms of the Creative Commons Attribution License, which permits use, distribution and reproduction in other forums, provided the original authors and source are credited and subject to any copyright notices concerning any third-party graphics etc. 


\section{APPENDIX}

A list of pseudo-utterances produced to convey each target emotion that were gated for presentation in the experiment.

1. I tropped for swinty gowers.

2. She kuvelled the noralind.

3. The placter jabored the tozz.

4. The moger is chalestic.

5. The rivix joled the silling.

6. The crinklet is boritate.

7. She krayed a jad ralition.

8. We wanced on the nonitor.

9. They pannifered the moser.

10. We groffed for vappy laurits.

11. I marlipped the tovity.

12. The varmalit was raffid.

13. They rilted the prubition. 